\title{
Proceso de creación de empresas de productos agroecológicos y consumo responsable en Yucatán
}

\author{
Rocío Blanco-Gregory \\ Universidad de Extremadura \\ rblanco@unex.es \\ Leonor López-Canto \\ Universidad Autónoma de Yucatán \\ lopez.canto@hotmail.es
}

Resumen: Frente al fenómeno de la globalización y a los evidentes estragos causados al medio ambiente y a las economías locales por las acciones depredadoras de las grandes corporaciones, ban surgido en las últimas décadas iniciativas en diferentes países que se presentan como una alternativa al paradigma predominante, basado en el modelo neoliberal, y que buscan impulsar el desarrollo desde un enfoque territorial mediante la recuperación de los espacios de intercambio económico por los actores locales, como estrategia para contribuir a la regeneración del tejido social.

Aun cuando existen diversos tipos de emprendimientos locales, en Yucatán, y dentro de una corriente de consumo conocida como slow food, desde el año 2008 se ha desarrollado un movimiento que agrupa a emprendedores locales que ofrecen de manera directa productos naturales a los que han incorporado, si bien en grados diferenciados, la visión de la sustentabilidad, es decir, productos agroecológicos.

Estas iniciativas no pretenden competir en los grandes circuitos productivos sino que ban encontrado un nicho de mercado, representado por un tipo de consumidores con un perfil diferente de los consumidores tradicionales, y que se inscriben dentro de la corriente del denominado «consumo responsable». 
Dentro de este contexto, resulta pertinente indagar acerca de las iniciativas emprendedoras de los productores que participan en el movimiento slow food en Yucatán, con el fin de tener un primer acercamiento a las características que distinguen este tipo particular de emprendimientos.

Por lo anterior, el objetivo de este trabajo es describir las características del proceso de creación de empresas, asi como identificar los factores clave que estimulan o inbiben las capacidades empresariales de los emprendedores pertenecientes al movimiento slow food en Yucatán.

Palabras clave: emprendimiento, productores agroecológicos, slow food, productos orgánicos, consumo responsable.

\section{Business creation process of agroecological products and responsible consumption in Yucatan}

Abstract: Faced with the phenomenon of globalization and the obvious damage caused to the environment and local economies by the predatory actions of large corporations, initiatives have emerged in different countries in recent decades which seek to provide an alternative to the predominant paradigm based on the neoliberal model. These alternatives take a territorial approach to development by recovering spaces that can be used for economic exchanges by local stakeholders and thus help to regenerate the social fabric.

Even though there are different types of local enterprise, as part of the Slow Food movement in Yucatan, since 2008 there has been a movement that brings together local entrepreneurs who offer directly natural products to those who have taken on to a greater or lesser extent the concept of sustainability- These products are known as agroecological products.

These initiatives are not intended to compete with mass production but they have found a niche market, in the form consumers who have an explicit interest in so-called "responsible consumption".

Consequently, the objectives of this paper are to describe the process of creating companies and to identify the key factors that stimulate or inbibit the entrepreneurial skills of entrepreneurs belonging to the Slow Food movement in Yucatán.

Keywords: entrepreneurship; agroecological producers; Slow Food; organic products; responsible consumption. 


\section{Planteamiento del problema y objetivos}

Frente a los profundos estragos causados a las economías regionales por la globalización, se ha desarrollado una corriente importante que enfatiza la necesidad de voltear la mirada nuevamente hacia las comunidades para impulsar el desarrollo regional, entendido como un fenómeno endógeno en el que interactúan fuerzas económicas, institucionales, políticas, geográfico-territoriales y culturales. Dos aspectos fundamentales en este tema son la creación de empresas y el individuo detrás de este proceso: el emprendedor.

Por otra parte, ante los daños irreparables al medio ambiente ocasionados por las grandes corporaciones, organismos de talla internacional como la Organización de las Naciones Unidas han planteado un nuevo paradigma de desarrollo: el desarrollo sustentable, lo cual a su vez conlleva de manera implícita una nueva concepción de consumo: el consumo responsable.

Dentro de una corriente de consumo conocida como slow food — surgida en Italia en 1986 como respuesta ante la dominación de cadenas de comida rápida, supermercados y agronegocios, y que evolucionó desde ser una asociación eminentemente gastronómica hasta convertirse en un movimiento político y social (Andrews, 2008), y entre cuyos objetivos está proteger el patrimonio alimentario de la humanidad-, presente en más de 130 países, entre ellos México, en Yucatán, desde el año 2010, se ha desarrollado un movimiento que agrupa a emprendedores locales que ofrecen de manera directa diversos productos alimenticios orientados al consumidor final, esto es, eliminando o reduciendo considerablemente la cadena de distribución. Entre ellos, se ha identificado a algunos que ofrecen productos del campo que han incorporado en sus procesos productivos, en diversos grados, la visión de la sustentabilidad, es decir, productos agroecológicos.

Estas iniciativas no pretenden competir con las grandes cadenas de supermercados, sino que han encontrado un nicho de mercado, representado por consumidores con poder adquisitivo, que buscan adquirir frutas y vegetales de la región cultivados sin fertilizantes químicos, así como otros productos de origen animal: carnes, huevos y leche, y otros elaborados de manera artesanal, como quesos, panes y confituras.

Dentro de este contexto, resulta pertinente indagar acerca de las iniciativas emprendedoras de los productores que participan en el movimiento slow food en Yucatán, con el fin de tener un primer acercamiento a las características que distinguen este tipo particular de emprendimientos, es decir, estudiar este movimiento desde el lado de la oferta.

Por lo anterior, el objetivo de este trabajo es describir las características del proceso de creación de empresas, así como identificar los factores clave que es- 
timulan o inhiben las capacidades empresariales de los emprendedores pertenecientes al movimiento slow food en Yucatán.

\section{Marco teórico}

\subsection{El emprendimiento}

El estudio de las personas que manifiestan comportamientos emprendedores cuyo resultado es la creación de valor agregado conforma el campo del emprendimiento $^{1}$ (Filion, 2001). El resultado (la creación de una empresa o el desarrollo de un proyecto) y el sujeto (el emprendedor) ${ }^{2}$ son inseparables.

Smallbone (2011, en Wennkers y Thurik) señala que el emprendimiento se refiere a lo siguiente:

La habilidad manifiesta y la voluntad de los individuos, bien sea solos o en equipo, dentro y fuera de organizaciones existentes, para, primero, percibir y crear nuevas oportunidades económicas (nuevos productos, métodos de producción, esquemas organizacionales y combinaciones de producto-mercado); y segundo, introducir sus ideas en el mercado, frente a la incertidumbre y otros obstáculos, tomando decisiones en cuanto a la localización, forma y uso de recursos e instituciones (Smallbone, 2011: 18).

Aunque en un sentido estricto el campo de estudio del emprendimiento está vinculado con la creación de nuevas empresas, aún entre los mismos investigadores especializados en el tema no hay consenso en los límites entre el campo de estudio del emprendimiento y el de la pequeña empresa (Smallbone, 2011; Kantis et alii, 2002; Bruyat, 1993). Siguiendo a Bruyat (1993), este identifica cuatro diferentes enfoques en la delimitación del campo, que incluyen zonas de convergencia y de divergencia: 1) como el estudio de la creación de una organización, 2) como el estudio del evento emprendedor, orientado generalmente al estudio de la pequeña empresa, 3) como sinónimo de innovación, y 4) como la creación de un valor nuevo importante, el cual se trata de una extensión del enfoque anterior.

Después de la perspectiva pionera de los economistas en el estudio de la actividad empresarial (Cantillon en 1755, Say en 1815 y Schumpeter desde 1928), un enfoque predominante a partir de la década de los sesenta del siglo pasado

1 El término anglosajón entrepreneurship y el francés entrepreneuriat han sido traducidos como emprendedorismo, emprendedurismo, empresarialidad, espíritu empresarial, espiritu emprendedor, etc., todos ellos para referirse al mismo campo.

2 El término entrepreneur a veces se ha utilizado como empresario y, en otras ocasiones, como emprendedor. Por citar un ejemplo, véase el documento en inglés de Kantis, Ishida y Komori (2002) y compárese con la versión en español. 
se orientó a tratar de descubrir los rasgos de personalidad que caracterizan a un emprendedor. Un enfoque más reciente consiste en estudiar el comportamiento de los emprendedores, qué actividades realizan y las razones por las cuales las hacen, aspectos íntimamente relacionados con el proceso de creación de empresas. Aunque se ha estudiado a los emprendedores dentro de un contexto de negocios, varios de los principios aplicables a dicho contexto — redes, movilización de recursos, representación empresarial - también son válidos para otro tipo de organizaciones que no son negocios (Aldrich, 1999).

\subsection{Emprendimiento social}

La literatura especializada distingue entre el emprendimiento tradicional, asociado a los negocios, y el emprendimiento social (Clamp y Alhamis, 2010). Este último es un campo de estudio muy reciente que emergió en el mundo académico a finales de los noventa en Estados Unidos y en el Reino Unido, aunque un antecedente más lejano se remonta a 1983, cuando Young escribió acerca de los «emprendedores innovadores no-lucrativos» bajo la línea schumpeteriana (Bacq y Janssen, 2011).

Los enfoques tradicionales (de economistas y estrategas) ven el emprendimiento como una respuesta racional a las presiones competitivas de los mercados, diseñado para generar beneficios a individuos u organizaciones. Sin embargo, estudios recientes han argumentado que el emprendimiento puede crear beneficios sociales, cuando las organizaciones e individuos se vuelven más sensibles y responsables hacia sus comunidades, lo que constituye el estudio del emprendimiento social.

En lugar de usar la creación de riqueza como medio para medir la creación de valor, la misión social es explícita y central para el emprendedor social. Para este emprendedor, la riqueza es usada solo como un medio para lograr un fin; la mayor parte de la utilidad económica generada por las actividades comerciales de la organización social es reinvertida en la misión social, mientras que en una empresa comercial tradicional las utilidades son distribuidas entre los propietarios, o reinvertidas en las actividades económicas de la compañía. En vez de movilizar recursos guiado por motivos de beneficio privado, un emprendedor social tiene un rol positivo en la regeneración cívica dentro de una comunidad en desventaja. Él puede mover recursos en áreas con baja productividad como una estrategia para fortalecer la economía, a través de la creación de empleos para los residentes locales, y atraer otros recursos de mercado, mediante subsidios públicos y filantrópicos (Clamp y Alhamis, 2010; Bacq y Janssen, 2011). 


\subsection{El proceso de creación de empresas}

El proceso de creación de empresas es un fenómeno complejo, contextualizado dentro de momentos y entornos específicos. En este fenómeno interactúan factores sociales, culturales y económicos (Aldrich, 1999; Klofsten, 2000; Filion, 2001; Ucbasaran, Gestead y Wright, 2001; Kantis et alii, 2002). La educación, la formación y los empleos anteriores del emprendedor, los valores del medio, la cultura familiar, etc., también juegan un papel importante (Filion, 2001).

Gibb y Ritchie, citados por Kantis et alii (2002), identifican las siguientes etapas en el proceso de creación de una empresa: la identificación de la idea, su validación, el acceso y organización de recursos, la negociación, el nacimiento y la supervivencia. El desarrollo exitoso del emprendimiento depende de cuatro factores clave: la idea en sí misma, la disponibilidad y obtención de recursos, la habilidad del emprendedor y sus asociados y el nivel de motivación y compromiso.

Kantis et alii (2002) definen tres etapas clave en dicho proceso: la gestación del proyecto, la puesta en marcha y el desarrollo inicial de la firma. Dentro de cada una de estas se pueden identificar determinados factores principales que inciden en los eventos críticos de cada etapa, los cuales se presentan en la imagen 1.

\section{Imagen 1. Proceso emprendedor}

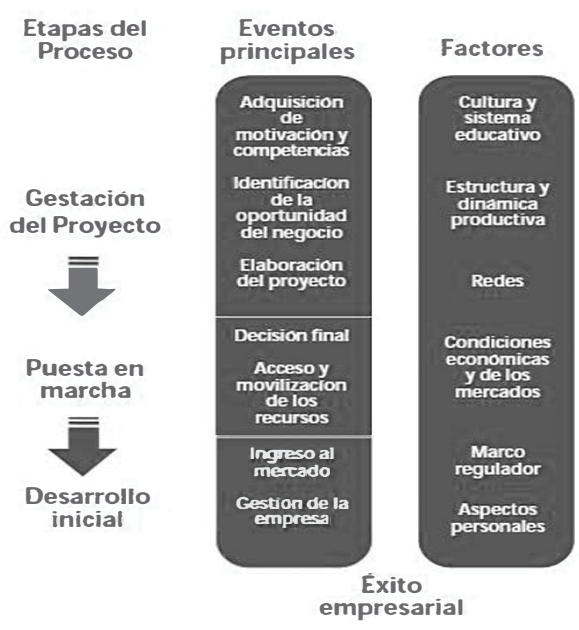

Fuente: Kantis et alii (2002).

Davies, Hides y Powell (2002) señalan que el crecimiento de pequeñas empresas exitosas hacia organizaciones de mayor tamaño implica múltiples cambios administrativos y organizacionales para sus creadores. Su habilidad para lidiar con estos cambios es crucial para la supervivencia y desarrollo de sus empresas. 


\subsection{El contexto}

La globalización constituye una nueva configuración espacio-territorial, entendiéndose el espacio (Mochi, 2006) como el soporte geográfico en donde se desarrollan las actividades socioeconómicas, mediante procesos que conllevan de manera implícita la idea de homogeneidad: unificación del mercado mundial, liberación comercial, papel de las comunicaciones, reducción de los costos de transporte, en donde la especificidad del territorio está permeada por las dinámicas nacionales e internacionales en las cuales participan las organizaciones e instituciones de la región (Riojas, 2007).

Boisier (2003: 14-16) propone un «hexágono del desarrollo», estableciendo seis grandes subsistemas locales existentes en cualquier sistema territorial -en el que hay que «establecer sinapsis, crear sinergia e introducir energía»-, uno de los cuales es precisamente el emprendedor, ubicado en el segundo subsistema. Estos subsistemas son los siguientes:

1) Un conjunto de valores: universales (libertad, democracia, justicia, solidaridad, paz, etc.) y singulares, propios del territorio en cuestión, que son los que le confieren su identidad propia.

2) Los actores, individuales, corporativos, colectivos, públicos y privados, quienes son los portadores del desarrollo. Aquí se debe distinguir entre actores en un sentido abstracto y agentes de desarrollo, portadores de proyectos y con poder efectivo para influir.

3) Las organizaciones públicas y privadas, subsistema cuyos elementos incluyen objetos, propiedades y conductas.

4) Los procedimientos, «conjunto de modalidades mediante las cuales el gobierno local gobierna, administra, informa y posiciona en el entorno a su propio territorio».

5) La acumulación o el capital económico.

Cada territorio específico es resultado, entonces, de procesos de estructuración que le han impreso su configuración actual: es un escenario en el cual se articulan y se tejen relaciones de poder, donde se conciben y se organizan iniciativas, donde juegan elementos como educación, cultura, historia, etc., que determinan o influyen en la capacidad percibida de sus habitantes para actuar. El territorio es en donde se manifiesta el emprendimiento en cualquiera de sus dos vertientes: la tradicional o la social. 


\subsection{El movimiento slow food en Yucatán}

El movimiento slow food se ha constituido en un actor con creciente presencia internacional, situándose en el marco de los debates actuales sobre el alcance de las redes de comidas alternativas y en los límites morales del mercado. Un creciente campo de investigación estudia a los consumidores, investidos de responsabilidades políticas, que responden a diferentes visiones de política y consumo, y de cultura y economía (Sassatelli y Davolio, 2010), lo cual detona sus decisiones de compra y de consumo. Dentro de este contexto, la aspiración del slow food de salvar las variedades, plantas y comidas amenazadas por la estandarización y homogenización de la agricultura resultante del amplio uso de prácticas convencionales (Lotti, 2008) ha ganado una creciente aceptación por parte de un público consumidor que apoya este movimiento, el cual está enfrentado con el paradigma prevaleciente de negocios, basado en el uso de la agricultura intensiva, con el consiguiente impacto negativo para la sociedad y el medio ambiente natural (Tencati y Zsolnai, 2012).

Lo anterior ha dado pie a la creación de un nuevo campo institucional, en donde el posicionamiento del movimiento slow food como un nuevo campo es resultado de la incorporación de nuevos actores y de la creación de una extendida identidad colectiva, lo cual ha hecho progresar al movimiento. El campo del slow food se ha transformado, de atraer solo a gastrónomos hasta volverse un campo más amplio que abarca activistas de justicia social y ambientalistas (Van Bommel y Spicer, 2011).

El movimiento Slow Food en Yucatán inicia en febrero de 2010, y agrupa a emprendedores locales que ofrecen de manera directa diversos productos alimenticios orientados al consumidor final, esto es, eliminando o reduciendo considerablemente la cadena de distribución. Entre ellos, se ha identificado a algunos que ofrecen productos del campo que han incorporado en sus procesos productivos, en diversos grados, la visión de la sustentabilidad, es decir, productos agroecológicos.

Este movimiento es el principal promotor del Mercado Fresco en la ciudad de Mérida, Yucatán, el cual agrupa a más de 53 productores que ofrecen sus productos todos los sábados en los alrededores de un conocido centro comercial de la ciudad (Diario de Yucatán, 29 de enero de 2017).

Según la misma fuente, hace diez años, un grupo de productores partidarios del movimiento, encabezados por Aliza Mishari Perkulis, abrió un pequeño mercado en un terreno alquilado en otro sitio de la ciudad de Mérida, iniciándose, de esta manera, la historia del Mercado Fresco; cuatro años después, se trasladaron al lugar que actualmente ocupan. En un principio, la mayoría de sus clientes eran 
extranjeros, aunque después comenzaron a ser visitados por consumidores locales, debido a que los productos ofrecidos eran cosechados en los huertos de los propios comerciantes y estaban libres de pesticidas y otros productos químicos. El pan, los quesos y la miel comercializados eran producidos de manera artesanal.

Aunque todos los productos son frescos, no todos ellos pueden ser denominados «orgánicos», pues para ello se requiere la certificación que otorga la SAGARPA $^{3}$ (Diario de Yucatán, 29 de enero de 2017), que consiste en el sello «ORGÁNICO SAGARPA MÉXICO», el cual brinda la certeza de ser un producto de calidad, sanidad y seguridad alimentaria (inocuidad) y garantiza a los consumidores que el producto cumplió con las normas mexicanas establecidas en la Ley de Productos Orgánicos (<http://www.sagarpa.gob.mx/Delegaciones/ coahuila/boletines/Paginas/2016B124.aspx $>$ ).

Los precios de los productos son un poco más altos, debido a que, al no utilizarse agroquímicos, en ocasiones las plantas mueren a causa de plagas que no pudieron combatirse, lo que ocasiona que el precio sea mayor, pero se tiene como beneficio que las verduras y frutas son frescas, más ricas en nutrientes y en sabor, además de que su cultivo no daña al medio ambiente (Diario de Yucatán, 29 de enero de 2017).

\section{Diseño y metodología}

El estudio llevado a cabo sobre la producción agroecológica en Yucatán ha sido de carácter cualitativo, realizando una serie de entrevistas en profundidad a los principales productores y comercializadores de estos productos etiquetados como orgánicos o cuya producción está basada en las características principales de la producción agroecológica anteriormente explicada.

Desde el punto de vista metodológico, la investigación cualitativa representa un modo específico de análisis del mundo empírico, que busca la comprensión de los fenómenos sociales desde las experiencias y puntos de vista de los actores sociales, y el entendimiento de los significados que estos asignan a sus acciones, creencias y valores. Como argumenta Izacara (2014), se trata de una metodología que se cimenta en la flexibilidad y la capacidad que ofrece al investigador, a la hora de analizar los procesos sociales que no son abordables a través de encuestas, de profundizar en una serie de elementos, procesos, significados, características y circunstancias que no se pueden medir a través de la cantidad, la frecuencia o la intensidad de los hechos (Ortí, 2000; Seale, 2002). En este sentido, creemos en la conveniencia e indispensabilidad de los discursos aportados por los testigos clave en primera persona, como ideólogos, creadores y ejecutores de sus iniciativas em3 Secretaría de Agricultura, Ganadería, Desarrollo Rural, Pesca y Alimentación. 
presariales, y, en suma, protagonistas de sus trayectorias particulares experimentadas haciendo uso de un emprendimiento social vinculado oportunamente al desarrollo incipiente del llamado movimiento Slow Food en el estado mexicano de Yucatán.

En concreto, se ha optado por la entrevista en profundidad, porque, siguiendo a Ortí (2000: 213), se trata de una técnica de investigación social que persigue la manifestación de los intereses informativos, las creencias y los deseos de los actores sociales; y, siguiendo a Izcara (2014), la entrevista indaga en los diferentes valores y significados que le atribuyen los informantes a los fenómenos sociales, y escudriña los significados subjetivos que para los entrevistados acarrea el hecho social investigado. De una forma más simple, la entrevista en profundidad podría definirse como un diálogo directo y espontáneo dirigido hacia la comprensión de las perspectivas que tienen los entrevistados respecto de sus propias experiencias (Taylor y Bodgan, 2000).

En esta investigación se realizaron 20 entrevistas en profundidad a productores y comercializadores del estado de Yucatán que exponen y venden sus productos los miércoles y los domingos en el llamado Mercado Slow Food en la capital del estado yucateco, en Mérida, a pesar de que sus producciones o negocios se encuentren en otras localidades del estado, más o menos cercanas a Mérida.

Dichas entrevistas, realizadas durante 2016 y 2017, fueron grabadas en soporte de audio y posteriormente fueron transcritas literalmente para su análisis en profundidad y la obtención de datos.

Tal y como se muestra en la siguiente tabla (tabla 1), entre los 20 informantes que participaron en las entrevistas en profundidad, nos encontramos con casos de emprendimiento de diferentes tipos, atendiendo a las siguientes variables: año de creación de la empresa, constitución legal del negocio, municipio, actividad empresarial principal del informante, género del emprendedor, principales productos que produce y/o comercializa, número de empleados que tiene la empresa y cifra de ventas semanales en pesos mexicanos. 
Tabla 1. Caracterización de los emprendedores agroecológicos entrevistados en Yucatán

\begin{tabular}{|c|c|c|c|c|c|c|c|c|}
\hline$\ddot{z}$ & 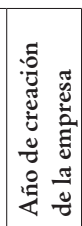 & 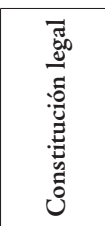 & 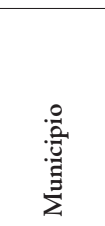 & 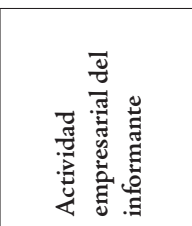 & 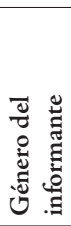 & 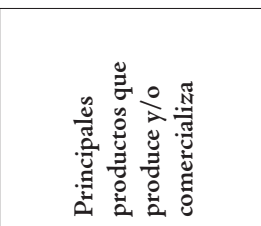 & 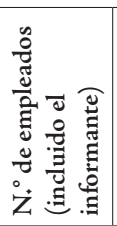 & 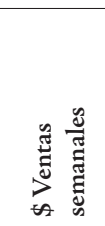 \\
\hline 1 & 2004 & $\begin{array}{l}\text { Sí - } \\
\text { Persona } \\
\text { física }\end{array}$ & Mérida & Comercializador & $\mathrm{F}$ & $\begin{array}{c}\text { Alimentos, corporal y } \\
\text { limpieza }\end{array}$ & 1 & $\begin{array}{c}\text { Más de } \$ \\
2.000\end{array}$ \\
\hline 2 & 2009 & No & Cholul & $\begin{array}{c}\text { Productor y } \\
\text { comercializador }\end{array}$ & $\mathrm{F}$ & $\begin{array}{l}\text { Tomate, huevo, ejote, } \\
\text { eneldo, arúgula, germi- } \\
\text { nados, frijol }\end{array}$ & $4-5(4)$ & $\begin{array}{c}\text { Más de } \$ \\
2.000\end{array}$ \\
\hline 3 & 1997 & $\begin{array}{l}\text { Sí- } \\
\text { SPR de } \\
\text { RL }\end{array}$ & Tizimín & Productor & M & $\begin{array}{c}\text { Cúrcuma, yuca, } \\
\text { jengibre, hoja de neem, } \\
\text { productos de medicina } \\
\text { alternativa }\end{array}$ & $2-3(3)$ & $\begin{array}{c}\$ 1.000- \\
2.000\end{array}$ \\
\hline 4 & 2014 & No & Mérida & $\begin{array}{c}\text { Productor y } \\
\text { comercializador }\end{array}$ & $\mathrm{F}$ & $\begin{array}{l}\text { Mermeladas, man- } \\
\text { tequilla de nueces y } \\
\text { semillas }\end{array}$ & $2-3(2)$ & $\begin{array}{c}\$ 1.000- \\
2.000\end{array}$ \\
\hline 5 & 2007 & No & Mérida & $\begin{array}{c}\text { Productor y } \\
\text { comercializador }\end{array}$ & M & $\begin{array}{c}\text { Tamales, comida } \\
\text { preparada }\end{array}$ & 1 & $\begin{array}{c}\text { Más de } \$ \\
2.000\end{array}$ \\
\hline 6 & 2012 & Sí & Mérida & $\begin{array}{c}\text { Productor y } \\
\text { comercializador }\end{array}$ & $\mathrm{F}$ & $\begin{array}{l}\text { Lechuga, arúgula, to- } \\
\text { mate, cilantro, fruta de } \\
\text { temporada y betabel }\end{array}$ & $4-5$ & $\begin{array}{c}\text { Más de } \$ \\
2.000\end{array}$ \\
\hline 7 & 2015 & No & Tecoh & $\begin{array}{c}\text { Productor y } \\
\text { comercializador }\end{array}$ & $\mathrm{F}$ & $\begin{array}{l}\text { Hojas de ensalada, } \\
\text { hierbas de temporada, } \\
\text { fermento japonés para } \\
\text { fertilizar, bioinsectici- } \\
\text { das y semillas }\end{array}$ & $2-3$ & $\begin{array}{c}\text { Más de } \$ \\
2.000\end{array}$ \\
\hline 8 & 2014 & $\begin{array}{l}\text { Sí- } \\
\text { Persona } \\
\text { física }\end{array}$ & Mérida & $\begin{array}{c}\text { Productor y } \\
\text { comercializador }\end{array}$ & M & $\begin{array}{l}\text { Cápsulas de moringa, } \\
\text { neem, corteza de neem, } \\
\text { graviola, cúrcuma y } \\
\text { alcachofa }\end{array}$ & $2-3$ & $\begin{array}{c}\text { Más de } \$ \\
2.000\end{array}$ \\
\hline 9 & 2015 & No & $\begin{array}{l}\text { Hunuc- } \\
\text { má }\end{array}$ & $\begin{array}{c}\text { Productor y } \\
\text { comercializador }\end{array}$ & M & $\begin{array}{c}\text { Papasul, pepita de } \\
\text { sikilpak, coco rallado, } \\
\text { chaya, espinaca y pitaya }\end{array}$ & $2-3$ & $\begin{array}{l}\text { Menos de } \\
\$ 1.000\end{array}$ \\
\hline 10 & 1997 & Sí & Mérida & comercializador & $\mathrm{F}$ & $\begin{array}{c}\text { Café orgánico, } \\
\text { productos de neem, } \\
\text { semillas, cereales, miel } \\
\text { y nutrientes }\end{array}$ & $2-3$ & $\begin{array}{c}\text { Más de } \$ \\
2.000\end{array}$ \\
\hline 11 & 2011 & Sí & Mérida & $\begin{array}{c}\text { Productor y } \\
\text { comercializador }\end{array}$ & $\mathrm{F}$ & $\begin{array}{l}\text { Pastas, salsas, beren- } \\
\text { jenas, conservas de } \\
\text { verdura. }\end{array}$ & $2-3$ & $\begin{array}{c}\text { Más de } \$ \\
2.000\end{array}$ \\
\hline 12 & 2011 & Sí & $\begin{array}{l}\text { Oxkutz- } \\
\text { cab }\end{array}$ & $\begin{array}{c}\text { Productor y } \\
\text { comercializador }\end{array}$ & $\mathrm{F}$ & $\begin{array}{c}\text { Miel abeja, harina de } \\
\text { yuca, jengibre y cúrcu- } \\
\text { ma en polvo, productos } \\
\text { frescos }\end{array}$ & $\begin{array}{l}\text { Más de } \\
5(8)\end{array}$ & $\begin{array}{c}\text { Más de } \$ \\
2.000\end{array}$ \\
\hline 13 & 2012 & $\begin{array}{l}\text { Sí- } \\
\text { SPR de } \\
\text { RL }\end{array}$ & Mérida & $\begin{array}{c}\text { Productor y } \\
\text { comercializador }\end{array}$ & M & $\begin{array}{c}\text { Hoja verde Stevia, } \\
\text { polvo molido Stevia y } \\
\text { mezclas de té }\end{array}$ & $\begin{array}{l}\text { Más de } \\
5(30)\end{array}$ & $\begin{array}{c}\text { Más de } \$ \\
2.000\end{array}$ \\
\hline
\end{tabular}




\begin{tabular}{|c|c|c|c|c|c|c|c|c|}
\hline 14 & 2011 & Sí & Mérida & $\begin{array}{c}\text { Productor y } \\
\text { comercializador }\end{array}$ & M & $\begin{array}{l}\text { Pan levadura madre, } \\
\text { pan sin gluten, pan } \\
\text { orgánico de semillas y } \\
\text { pretzel }\end{array}$ & $4-5$ & $\begin{array}{c}\text { Más de \$ } \\
2.000\end{array}$ \\
\hline 15 & 2011 & Sí & Mérida & Comercializador & $\mathrm{F}$ & $\begin{array}{c}\text { Huevo, cereales, } \\
\text { endulzantes, cuidado } \\
\text { personal y café }\end{array}$ & $4-5$ & $\begin{array}{c}\text { Más de \$ } \\
2.000\end{array}$ \\
\hline 16 & 2014 & No & Caucel & $\begin{array}{c}\text { Productor y } \\
\text { comercializador }\end{array}$ & $\mathrm{F}$ & $\begin{array}{c}\text { Hamburguesas de } \\
\text { lenteja y germinados, } \\
\text { camote, yuca, galletas } \\
\text { para perros y brownies } \\
\text { crudiveganos }\end{array}$ & 1 & $\begin{array}{l}\$ 1.000 \\
2.000\end{array}$ \\
\hline 17 & 2014 & Sí & Izamal & Comercializador & M & $\begin{array}{l}\text { Fermentos, cambucha } \\
\text { (probióticos), kéfir y } \\
\text { pan de cebolla }\end{array}$ & $2-3$ & $\begin{array}{c}\text { Más de } \$ \\
2.000\end{array}$ \\
\hline 18 & 2013 & Sí & Tepakán & $\begin{array}{c}\text { Productor y } \\
\text { comercializador }\end{array}$ & M & $\begin{array}{c}\text { Rulo de cabra, panela, } \\
\text { manchego y yogurt }\end{array}$ & $\begin{array}{l}\text { Más de } \\
5(7)\end{array}$ & $\begin{array}{c}\text { Más de } \$ \\
2.000\end{array}$ \\
\hline 19 & 2010 & Sí & Cholul & $\begin{array}{c}\text { Productor y } \\
\text { comercializador }\end{array}$ & M & $\begin{array}{c}\text { Huevo, semillas, abo- } \\
\text { nos, kale y arúgula }\end{array}$ & $4-5$ & $\begin{array}{c}\text { Más de \$ } \\
2.000\end{array}$ \\
\hline 20 & 2009 & Sí & Mérida & Comercializador & $\mathrm{F}$ & $\begin{array}{l}\text { Leguminosas, miel } \\
\text { API, semillas }\end{array}$ & 1 & $\begin{array}{c}\text { Más de } \$ \\
2.000\end{array}$ \\
\hline
\end{tabular}

Fuente: elaboración propia.

\section{Principales resultados}

A partir de la transcripción de las entrevistas en profundidad que se han realizado a los diferentes emprendedores de productos agroecológicos, se presentan ahora los resultados obtenidos sobre las cuestiones planteadas en el guion estructurado que se diseñó para dichas entrevistas, destacando la información cualitativa, por una parte, y los datos cuantitativos obtenidos de algunas preguntas que fueron susceptibles de ser cuantificados, por otra.

Los resultados se presentan de forma estructurada atendiendo a las diferentes partes en las que se diseñó el guion de la entrevista con respecto al objeto de este artículo, distinguiendo entre las dos partes en las que se divide el proceso de emprendimiento, explicado en el marco teórico: el proceso de emprender y crear la empresa, por un lado, y las etapas en el proceso de creación de la empresa, por otro, haciendo referencia entre estas últimas, como se verá más adelante, a la gestación, la puesta en marcha y el desarrollo inicial de la empresa.

\subsection{El proceso de emprender y crear la empresa}

En primer lugar, hay que señalar que, en cuanto al proceso de emprendimiento y creación de la empresa, a excepción de tres de los informantes que ya llevan una dilatada experiencia a sus espaldas en el asunto de la producción agroecológica, la mayoría de estos empresarios se iniciaron entre los años 2007 y 2015, y mayori- 
tariamente a partir de 2010, que es el año en el que se inicia el movimiento Slow Food en Yucatán.

Hacia 2016, solo seis de nuestros informantes no tienen sus empresas constituidas legalmente, ya sea en la modalidad autónoma o en forma de sociedad. Mayoritariamente este tipo de emprendimientos están dedicados a las dos actividades principales en el mercado: la producción y la comercialización, pero, a pesar de que la actividad comercial se concentra en la ciudad de Mérida, muchos de nuestros informantes, casi la mitad, poseen sus producciones en otros municipios de entornos más rurales cercanos a Mérida, como son Cholul, Tizimín, Caucel, Izamal, Tepakán, Oxkutzcab, Hunucmá o Tecoh, todos ellos pertenecientes al estado de Yucatán.

Existen casos de emprendimientos más desarrollados y de gran amplitud en su actividad para los que cuentan con un número importante de empleados, pero la media de empleados (incluido el empresario/emprendedor) se encuentra en torno a tres en este tipo de iniciativas, algo lógico si pensamos que gran parte de estos negocios tienen una trayectoria empresarial relativamente pequeña.

Respecto al volumen de ventas semanal de estos emprendimientos, la gran mayoría supera los 2.000 pesos mexicanos semanales.

Mayoritariamente la producción y comercialización de productos agroecológicos se centran en los sectores agrícola y ganadero, teniendo más representación la agricultura, en todo caso, aunque también se están desarrollando los productos para la salud e higiene corporal y para la limpieza doméstica.

\subsection{Etapas en el proceso de creación de la empresa}

En el proceso de creación de empresa, a la hora de elaborar el guion estructurado de la entrevista a la que se ha sometido a nuestros informantes, distinguimos tres etapas, siguiendo el modelo de Kantis et alii (2002): gestación de la empresa, puesta en marcha y desarrollo inicial.

\subsubsection{Gestación de la empresa}

Las principales motivaciones que se han encontrado en estos emprendedores, a la hora de pensar en la creación de su negocio, son diferentes, y en algunos son más importantes unas, y en otros, otras diferentes, pero, en general, se podrían destacar las que más profundamente han contribuido a esta idea de gestar sus propios negocios: para contribuir a la sociedad, para contribuir a generar hábitos saludables en la sociedad, por creer en la necesidad de preservar el medio ambiente y para lograr una realización personal. Es cierto que no estamos hablando de emprendimientos sin ánimo de lucro, pero la mayoría de nuestros informantes no lo 
han hecho fundamentalmente para ganar más dinero ni para ser sus propios jefes o por el hecho de que esté de moda en la actualidad, como se puede apreciar en el siguiente gráfico (gráfico 1).

\section{Gráfico 1. Principales motivos de los emprendedores cuando pensaban en la gestación de la empresa}

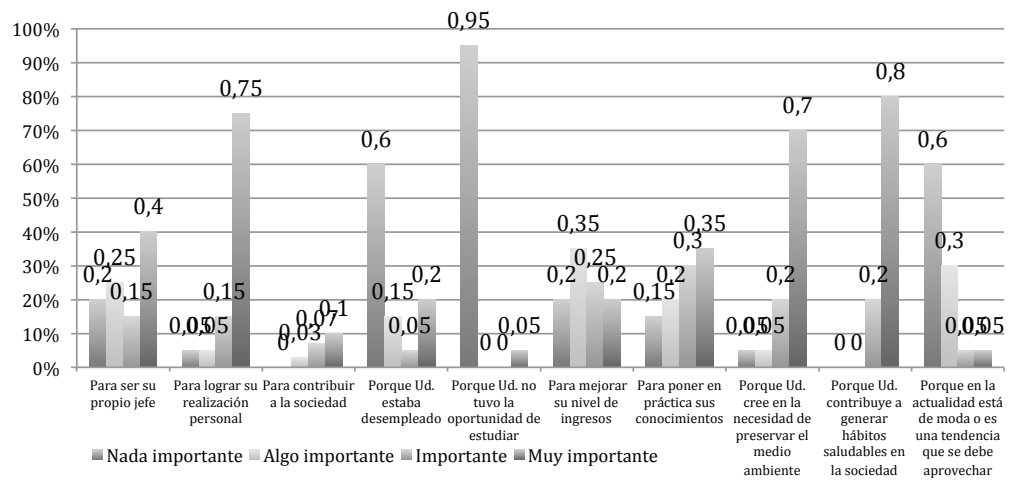

Fuente: elaboración propia a partir de las entrevistas realizadas.

En cuanto a la reacción, por parte de los diferentes grupos de socialización con los que interactúan estos emprendedores (familia, amigos, colegas de profesión, profesores, etc.), al conocer su decisión de crear una empresa de este tipo, hemos de señalar que ha sido diversa en los diferentes informantes. Nos encontramos con un apoyo familiar por parte de quien ya ha tenido antecedentes familiares de emprendimiento o bien comparten el negocio con su pareja o la familia:

E13: Mi padre fue empresario y me impulsó.

E11: Bien. Esa fue la decisión que tomamos juntos. Yo trabajo con mi esposo, desde siempre, por eso tomamos la decisión juntos.

En otros casos, el apoyo de la familia ha sido negativo y, en cambio, el de los colegas o profesores ha sido más positivo:

E8: Lo comenté con unos doctores porque hasta cierto punto es de grado medicinal y conté con el total apoyo de ellos.

E19: Bueno, mi maestra de agricultura dijo que estaba genial.

Otros, por ejemplo, aluden a que no se puede responder a esta pregunta ya que el negocio no se gestó con una idea de él como tal, sino como una cuestión de autoconsumo y como actividad de entretenimiento: 
E2: Mira, nada más te voy a poner en contexto, nosotros nunca pensamos que esto iba a ser un negocio, yo regresé de la escuela de Maní, le dije a mi esposo «hazme un gallinero porque quiero unas gallinitas para consumo local», y, este, «hazme unas parcelas porque quiero cultivar para nosotros»; esto empezó así, al año siguiente en lugar de cinco gallinas tenía 30, a los tres años tenía 100 y ahorita tengo 500. No lo pensamos, no se planeó; empezamos pensando que tenemos que comer de este jardín que era demasiado grande.

Con respecto a la oferta de los productos orgánicos en el mercado yucateco, si bien sabemos que tratamos de un asunto relativamente nuevo, donde su desarrollo se empieza a percibir a partir de hace, escasamente, unos años, los entrevistados manifiestan en su mayoría (aunque dependiendo del tipo de productos) que sus productos no se ofertaban en el Estado de Yucatán y que, o bien no existía demanda por desconocimiento de la población (E10ः «No, de hecho decían si era café orgásmico») o apenas existía una demanda incipiente, pero que en algunos productos, poco a poco, iba creciendo:

E12: Había una demanda incipiente. Poquito a poquito se iba interesando la gente en eso. Te puedo decir que los primeros clientes fueron el CAT de TELCEL (los teléfonos celulares). Tenía una amiga allá que sabía que teníamos productos orgánicos y les contaba a todos sus amigos y me hacían pedidos, ¿no? Medio kilo de limón, medio de no sé qué, medio de lo otro..., entonces yo llegaba a la salida del trabajo de ellos con la cajuela llena y así a repartir todo lo que me pedían. Y así empezó hasta que de repente me dijeronः «Oye, ¿facturas y esto?». Y la miel, por ejemplo, porque es de los más fuerte, pues bueno, porque hay que constituirlo legalmente. Y las tiendas. Mucho fue también por las tiendas. Por ejemplo, Carolina fue una de las primeras clientes; YA AXTAL fue la primerísima, creo, con M. ${ }^{a}$ Carmen. Y de repente, en ese «si facturas no facturas; he oído hablar de ti...», ya fue cuando constituimos la empresa legalmente.

A continuación, nos pareció interesante indagar en las fuentes que contribuyeron en nuestros informantes al proceso de identificar la idea de negocio que ellos tenían en mente y podemos comprobar, en este caso, que de los $20 \mathrm{em}$ prendedores que fueron entrevistados, para la mayoría (17), la principal fuente consistió en la interacción social con otras personas, preguntando y resolviendo las dudas que suelen aparecer en esta fase de la creación de un negocio. La sigue, muy de cerca, la consulta en Internet (16), asistir a ferias comerciales (13) y leer artículos académicos al respecto (13).

También, en esta etapa, se quiso conocer el nivel de conocimiento que tenían los emprendedores sobre ciertos aspectos considerados muy importantes para la puesta en marcha de sus empresas, ya haya sido en forma de comercialización o 
Gráfico 2. Fuentes que contribuyeron a identificar la idea de negocio de los emprendedores agroecológicos

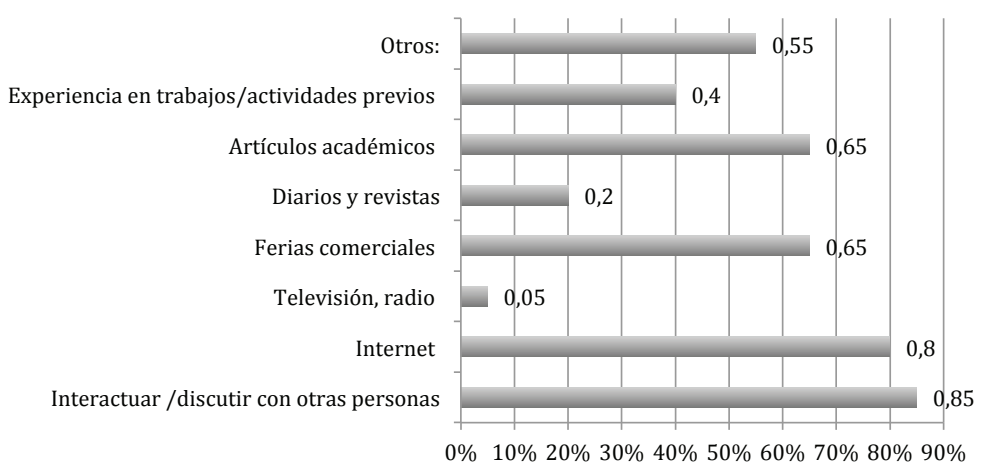

Fuente: elaboración propia a partir de las entrevistas realizadas.

de producción de los productos agroecológicos. La mayoría de ellos desconocían la inversión necesaria para poner en marcha sus negocios (15), y también cómo era el tamaño del mercado en el que se disponían a emprender (14). En cambio, sí poseían conocimientos técnicos sobre sus negocios y cómo acceder a ellos (15), e incluso conocían la cantidad de competidores que tenían y qué características tenían (15), cuestiones todas ellas muy relevantes a la hora de introducirse y posicionarse en un mercado por primera vez (gráfico 3).

Gráfico 3. Información de los emprendedores agroecológicos sobre los siguientes ítems antes de iniciar su empresa

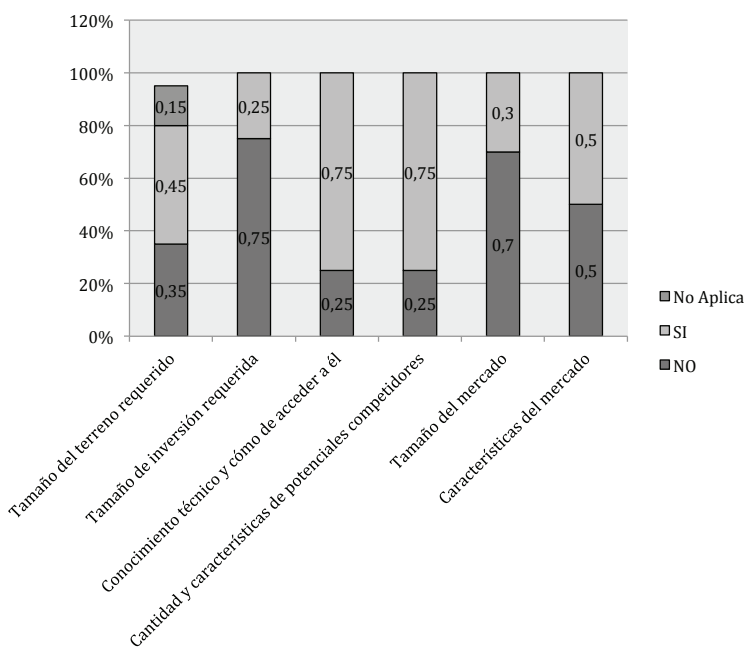

Fuente: elaboración propia a partir de las entrevistas realizadas. 
Finalmente, en esta primera etapa, también nos pareció significativo — como paso a seguir en un proceso de emprendimiento de una empresa - averiguar cuántos de nuestros informantes, previamente a tomar la decisión de crear su propio negocio, realizaron un plan de empresas, hicieron una estimación aproximada de las ventas y de los costes que iban a tener o calcularon los ingresos que esperaban obtener en el futuro. Y nos encontramos, sorprendentemente, con el hecho de que 15 de los 20 informantes no han realizado previamente un plan de empresas y solo la mitad de ellos calcularon aproximadamente las ventas y los costes que iban a tener, así como los ingresos que esperaban obtener en un futuro.

Gráfico 4. Decisión, por parte del emprendedor, de realizar alguno de los siguientes estudios para tomar la medida final de iniciar el negocio

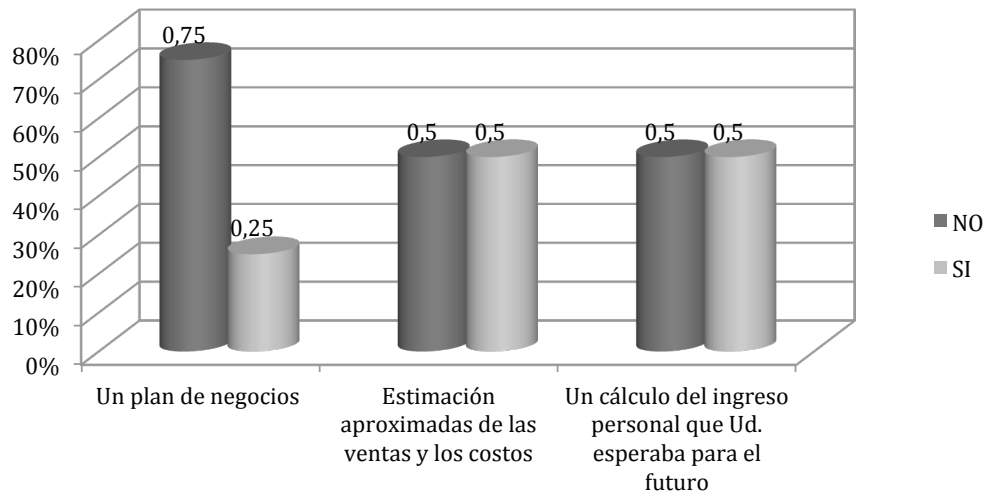

Fuente: elaboración propia a partir de las entrevistas realizadas.

\subsubsection{Puesta en marcha de la empresa}

En esta segunda etapa, tratamos de indagar sobre las fuentes de financiación de las cuales han necesitado hacer uso los emprendedores para poner en marcha sus empresas. Por una parte, hemos distinguido entre fuentes internas: ahorros personales, recurrir a la ayuda de familiares y amigos o hacer uso de la tarjeta de crédito personal. Por otra, fuentes externas: préstamos bancarios, préstamos de instituciones públicas y subsidios de instituciones públicas. Y, por último, se ha hecho referencia a otras fuentes, como el atraso de pago de impuestos, de servicios o salarios a los empleados y otras fuentes de financiación que los emprendedores hubieran podido utilizar y no se encuentran contempladas entre las ya mencionadas.

Si se observa el gráfico 5, comprobamos que la mayoría de los entrevistados han recurrido, tanto al inicio de la puesta en marcha del negocio como en los 
primeros años de vida de este, fundamentalmente, a fuentes internas de financiación: sobre todo a ahorros personales, $y$, en menor medida, han recurrido a familiares y amigos y a la tarjeta de crédito personal. Las fuentes de financiación externas apenas han sido utilizadas por nuestros informantes y alrededor de una cuarta parte de ellos han tenido que hacer uso de otras fuentes (tanto al inicio como en los primeros años), como pueden ser el retraso de pagos de impuestos, de servicios o de salarios a los empleados. Así lo manifiestan literalmente algunos de los entrevistados:

E2: Quise tener pero, pero no, nunca nos dieron nada, hicimos todo el trámite se hizo todo por ahí, un año yendo al Gobierno, no se hizo nada. Se trataba de fondos perdidos que los daba la mitad el Gobierno federal y la otra, el estatal.

E17: Estoy persiguiendo subsidios de instituciones públicas pero no lo he conseguido.

E19: Al inicio recibí un apoyo de 30.000 pesos y ya. Fue el único subsidio que recibí. Y otra vez gané un concurso en un documental y ya, pero fueron las únicas dos cosas que recibí. Nunca he solicitado ni apoyo, ni beca, ni financiamiento o subsidio.

E20: Otros: la liquidación de mi trabajo anterior.

\section{Gráfico 5. Fuentes de financiación utilizadas por los emprendedores para la puesta en marcha de sus empresas, tanto al inicio como en los primeros años}

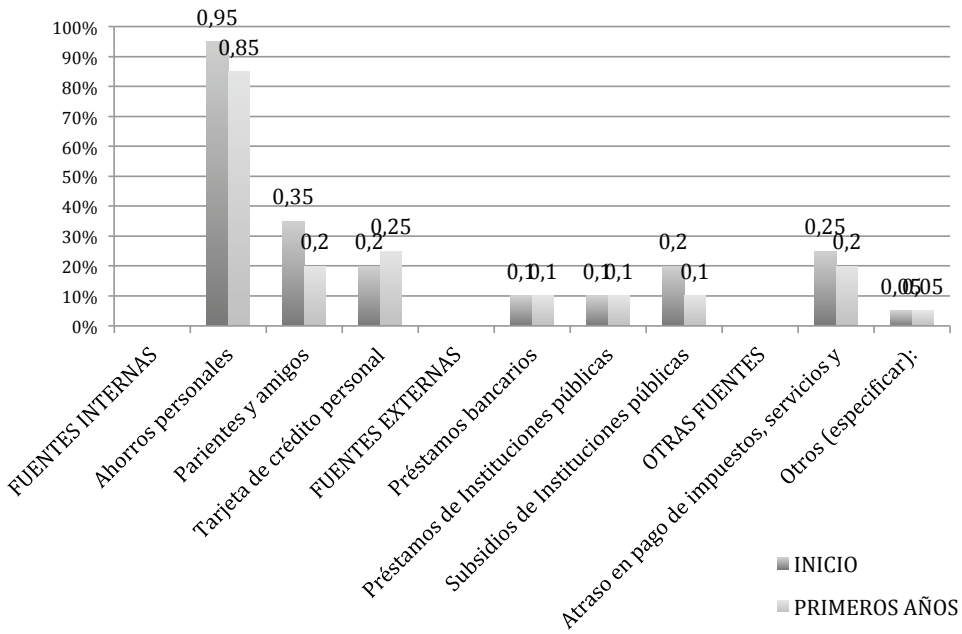

Fuente: elaboración propia a partir de las entrevistas realizadas. 


\subsubsection{Desarrollo inicial de la empresa}

Los principales problemas a los que se enfrentan los emprendedores en los tres primeros años de vida de la empresa, y algunos de ellos aún no se han resuelto, son de diferente índole. De todo el elenco que figura en el gráfico 6, los más destacados por los entrevistados convergen en los cinco siguientes: conseguir clientes, tener un flujo de efectivo balanceado, conseguir proveedores adecuados, certificar con estándares de calidad y contratar empleados cualificados. Algunos de estos problemas ya se han resuelto en la mayoría de los emprendedores entrevistados, pero hay otros que todavía persisten en una gran mayoría de ellos, fundamentalmente los cuatro últimos mencionados anteriormente, ya que conseguir clientes parece que se presenta como un problema común al principio, pero a día de hoy casi todos los emprendedores analizados ya han solventado ese problema.

\section{Gráfico 6. Principales problemas a los que se enfrentan los emprendedores en los tres primeros años de la empresa y resolución de estos}

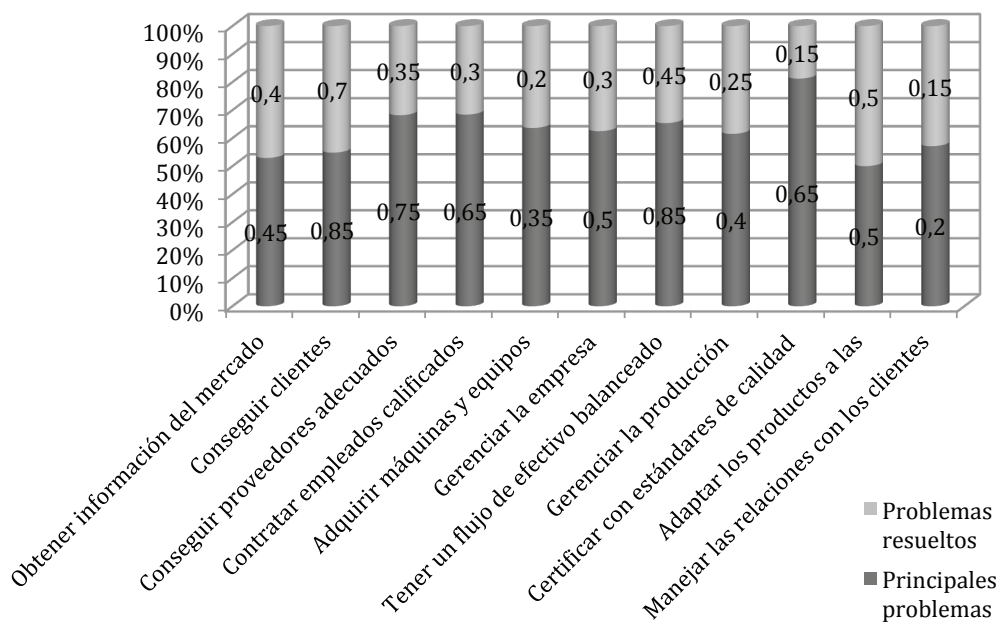

Fuente: elaboración propia a partir de las entrevistas realizadas.

$\mathrm{Al}$ respecto, señalamos algunas de sus manifestaciones que nos parecen muy significativas:

E1 (conseguir proveedores adecuados): Pero ¿sabes qué fenómeno ha pasado? Que si se abre una tienda nueva da este giro pero es moda o trae cosas internacionales, el mercado meridano se va y luego regresan, y te dicen que no venían por irse y el proveedor igual, porque me sigue pasando, de repente te deja mal, le prefiere 
dar a otra tienda que a ti, creo que ese es un día a día. Sí, los tienes que estar solucionando. Nunca dejas de...

E2 (tener un flujo de efectivo balanceado): Eso siempre ha sido un problema y sigue siendo en todo México.

E3 (conseguir clientes): No es un problema que resulte..., déjame, te explico cómo es. Como es una empresa pequeña, tenemos un mercado local muy..., es un nicho de mercado muy especial, cada vez más gente conoce mis productos, pero..., avanza, pero muy lento, porque no puedo pagar una campaña de publicidad o algo así, entonces...

E5 (certificación de calidad): Pues digamos que yo solo soy el que certifica mis propios productos, bueno, de alguna forma el producto es el que habla y da la cara. Entonces, con eso de las exigencias en cuanto a las normas de calidad..., como no estoy dado de alta entonces nunca me han dicho nada.

E7 (certificación de calidad): No comulgo con esa bandera, sin embargo, sé que en algún punto vamos a tener que entrar por lo menos en certificaciones comunitarias.

E18 (certificación de calidad): No me interesa. Nunca me importó. Lo que sí me gustaría es que los productos fueran evaluados nutricionalmente con una tabla de valores. Eso sí es para mí importante. Llevarlo a la Química de la UADY y que te hagan estudios sobre grasa, humedad, proteínas...

E19 (certificación de calidad): No, mis estándares personales de calidad son buenos [risas].

E18 (contratar empleados cualificados): Sí. Absolutamente problemático. Y sigue siendo un problema. Ahí yo lo subrayaría con tres rayas. Es que Yucatán es uno de los lugares que en el área del campo es muy complicado encontrar a gente cualificada y afecta realmente al emprendimiento. Es casi un tema que en un momento determinado de planteas cambiar de lugar ${ }_{.+.}$¡Así te lo digo!

E19 (adaptar los productos a las necesidades de los consumidores): Obligamos a la gente a que coma lo que sembramos, o sea, educamos a la gente, ja ja ja. 


\subsection{Opinión de los emprendedores agroecológicos sobre el consumo y la demanda de productos slow food en su entorno}

En este estudio también nos pareció relevante conocer qué opinión tiene los emprendedores (ya sean productores o comercializadores) sobre el público objetivo, o, lo que es lo mismo, sobre los consumidores de dichos productos dentro de su entorno más próximo, es decir, en el estado de Yucatán, y, en concreto, en Mérida y en los municipios de alrededor. En el guion estructurado de la entrevista a la que se sometieron los informantes se incorporaron dos cuestiones sencillas pero que, a nuestro modo de ver, pueden explicar la novedad de este movimiento de consumo responsable y las motivaciones de los emprendedores a la hora de idear sus negocios y ponerlos en marcha como una forma de emprendimiento social, y los aspectos que este conlleva, explicados anteriormente. Una cuestión responde a la percepción de los emprendedores sobre la demanda de sus productos en el estado yucateco y la otra aborda una serie de cuestiones referidas al incipiente consumo apriorístico que suponíamos y donde se intenta conocer la opinión de los informantes para que sus empresas alcancen un gran desarrollo y se fomente el consumo de este tipo de productos frente a los cultivados tradicionalmente.

En cuanto al nivel de demanda que perciben en los consumidores (gráfico 7), hay que señalar que una gran mayoría, 14 de 20 empresarios entrevistados, indican que este es medio e incipiente, que falta aún mucha cultura del consumo de dichos productos; tan solo tres de los informantes piensan que la demanda, por parte del público, es alta y una proporción similar de ellos opinan que es muy baja.

Gráfico 7. Opinión de los emprendedores sobre el nivel de demanda de productos ecológicos en su entorno más próximo

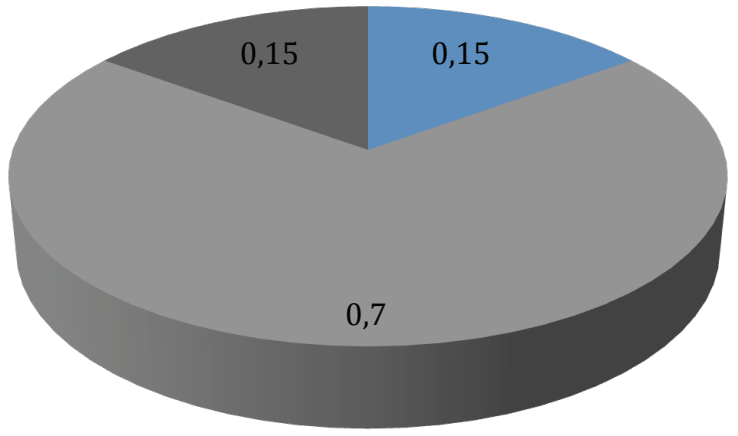

Muy bajo

Bajo

Medio

alto

Muy alto

Fuente: elaboración propia a partir de las entrevistas realizadas. 
Posteriormente, se quiso indagar sobre el grado de acuerdo o de desacuerdo en el que se situaban los entrevistados con algunas afirmaciones que les planteábamos en torno al consumo de dichos productos por parte del público consumidor del entorno. De los tres enunciados que les planteamos (véase gráfico 8), una mayoría significativa concordaba en que el motivo de que la demanda de dichos productos sea baja es por la falta de formación y de información en los consumidores y en los propios productores, considerándose la formación y la información - tanto a consumidores como a empresarios - unas herramientas cruciales e indispensables para que este mercado funcione.

\section{Gráfico 8. Opinión de los emprendedores sobre ciertas cuestiones relacionadas con la demanda de los productos orgánicos}

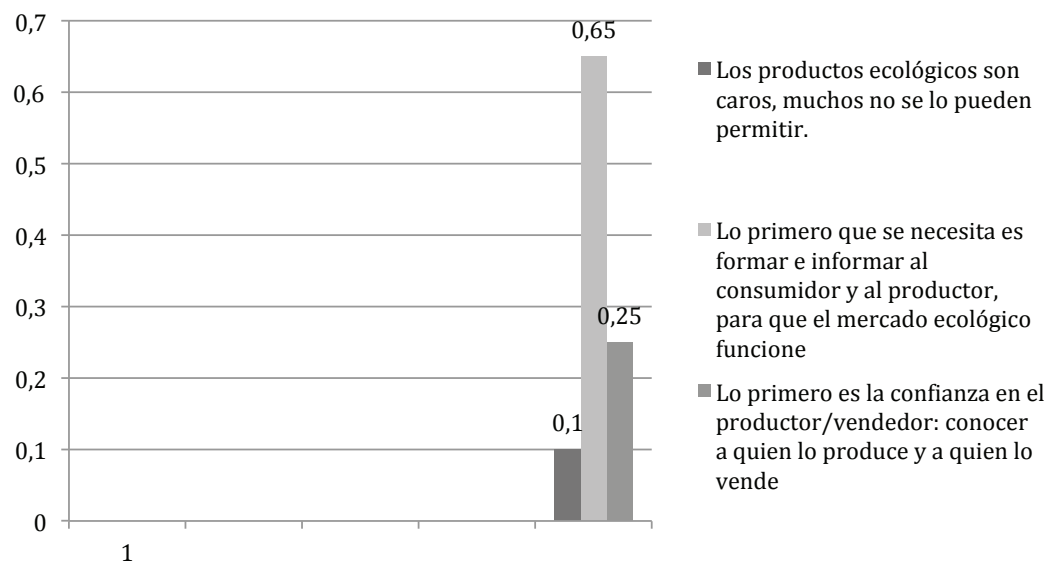

Fuente: elaboración propia a partir de las entrevistas realizadas.

\section{Conclusiones}

A raíz de esta investigación, se pone de manifiesto, una vez más, que las economías regionales y locales han sufrido graves deterioros debidos al proceso de globalización internacional, viéndose aún más afectadas aquellas economías que se identifican en los países menos desarrollados. Por tanto, con vistas a impulsar nuevamente estas economías locales de las comunidades menos desarrolladas, es necesario implantar medidas que promuevan y faciliten la generación de nuevos emprendedores y la creación de empresas que contribuyan a la dinamización de dichas economías, en aras de preservar e impulsar los productos autóctonos y los sistemas de producción y consumo, en este caso, de carácter saludable para la sociedad y orientados hacia la preservación del medio ambiente. En el estado 
mexicano de Yucatán, poco a poco, están emergiendo nuevos procesos de emprendimiento referidos a la producción y comercialización de productos agroecológicos donde los emprendedores se están abriendo camino y dando a conocer este tipo de producción natural y responsable con el medio ambiente en forma de productos agroecológicos y desarrollando iniciativas que sirvan de contexto para dar a conocer y comercializar sus productos frescos, como el llamado Mercado Slow Food de Mérida (capital del estado), que, apoyado en el movimiento ecologista conocido por el mismo nombre, está sirviendo de sostén y empuje a estas iniciativas emprendedoras.

En la investigación al respecto, se ha desarrollado un estudio de carácter cualitativo para conocer el nivel y las características de los nuevos y pequeños emprendedores que están apareciendo en el estado de Yucatán, específicamente, en los territorios cercanos a la capital, Mérida, donde se concentra la mayor parte de la actividad empresarial, teniendo en cuenta que los sectores agrícola y ganadero son los que tienen más presencia en la mayoría de los emprendimientos de carácter agroecológico u orgánico. En dichos procesos de emprendimiento, hay que destacar que sobresale un tipo de emprendimiento motivado por su carácter social, distinto al emprendimiento tradicional que estaba fundamentalmente motivado por el hecho de realizar negocios con fines exclusivamente lucrativos.

Estos tipos de emprendimiento enfocados en producciones agrícolas y ganaderas - fundamentalmente - han sido desarrollados y motivados por ideologías y convicciones naturalistas de sus emprendedores y empiezan a tomar fuerza, asociados al nacimiento de movimientos ecologistas, como el conocido movimiento slow food, con un marcado carácter de sostenibilidad y preservación del medio ambiente, además de la preocupación por una alimentación saludable, incidiendo, por tanto, en sistemas de producción y comercialización de tipo agroecológico.

Entre los emprendedores de productos agroecológicos en Yucatán, encontramos indistintamente a hombres y a mujeres, tratándose de iniciativas relativamente jóvenes, pues la gran mayoría de las empresas analizadas se crean a partir de 2010, coincidiendo con los inicios del movimiento slow food. En su mayoría, se trata de pequeñas sociedades constituidas legalmente, aunque casi un tercio de ellas aún no han conseguido su registro legal, destacándose como actividades prioritarias en dichas empresas la agricultura, en primer lugar, y la ganadería, en segundo puesto. Y, además — debido a su corta trayectoria, por lo general — estas pequeñas sociedades cuentan con una media de tres empleados (incluido el propio emprendedor), facturando, una gran mayoría de los emprendedores entrevistados, por encima de los 2.000 pesos mexicanos semanales. 
Con respecto a las etapas que se han seguido en el proceso de creación de la empresa, se han distinguido tres momentos fundamentales en la trayectoria de los emprendedores que han sido entrevistados: la gestación de la empresa, la puesta en marcha y su desarrollo inicial.

Al estudiar la gestación de la empresa, en los diferentes casos, hay que destacar que entre los emprendedores entrevistados sobresalen, entre muchos, cuatro motivos fundamentales que les llevaron a pensar en su idea de negocio: para contribuir a la sociedad, para contribuir a generar hábitos saludables en la sociedad, por creer en la necesidad de preservar el medio ambiente y para lograr una realización personal. Por otra parte, se debe apuntar también que el apoyo que han recibido por parte de las personas más próximas de su entorno no ha sido unánime en todos ellos, viéndose algunos más arropados que otros por la familia, los amigos, etc.

En esta misma fase y como variable fundamental a la hora de idear un negocio, hay que tener una visión clara sobre la oferta de productos similares en el mercado. Y a propósito de ello, los emprendedores manifiestan que sus productos no se ofertaban en el estado de Yucatán y que no existía ninguna demanda o existía una demanda muy sutil que empezaba a desarrollarse. Para la mayoría de ellos, se destacan como fuentes principales que contribuyeron al proceso de identificación de su idea de negocio la interacción social con otras personas (preguntando y resolviendo las dudas que suelen aparecer en esta fase de la creación de un negocio), la consulta en Internet, asistir a ferias comerciales y la lectura de artículos académicos al respecto.

Destacamos que nos encontramos con emprendedores que están formados y tienen conocimientos sobre la actividad a realizar y la competencia en el mercado, pero, en cambio, desconocen aspectos más economicistas y propios de un empresario, como son el tamaño del mercado en el que han decidido emprender y la inversión necesaria para la puesta en marcha del negocio. De hecho, tres cuartas partes de los entrevistados no han realizado previamente un plan de empresas y solo la mitad de ellos han realizado un cálculo aproximado de las ventas y los costes que van a tener, y de los ingresos que esperan obtener en un futuro.

Con respecto a la segunda etapa, la puesta en marcha, la mayoría de los entrevistados han recurrido, tanto al inicio como en los primeros años de vida de su empresa, a fuentes internas de financiación, destacando ahorros personales y, en menor medida, el recurso a familiares y amigos y a la tarjeta de crédito personal. Alrededor de una cuarta parte de ellos han hecho uso de otras fuentes (tanto al inicio como en los primeros años), como el retraso de pagos de impuestos, de servicios o de salarios a sus empleados. 
Ya en la etapa del desarrollo inicial de la empresa, hay que destacar que los principales problemas a los que se han enfrentado y se siguen enfrentando los emprendedores en los tres primeros años de vida de la empresa son muy diversos: conseguir clientes, tener un flujo de efectivo balanceado, conseguir proveedores adecuados, certificar con estándares de calidad y contratar empleados cualificados.

Finalmente y para concluir este estudio, debemos resaltar la opinión que tienen los emprendedores sobre su público objetivo o los consumidores de dichos productos dentro de su entorno, destacando dos aspectos importantes: la incipiente demanda por parte de los consumidores con escasa cultura de consumo de productos agroecológicos y la falta de formación y de información en los consumidores y en los propios productores, considerándolo el principal motivo de que la demanda de dichos productos sea baja y de que este mercado de productos agroecológicos u orgánicos no termine de funcionar a pleno rendimiento.

\section{Limitaciones y futuras líneas de investigación}

Por cuestiones de tiempo y disponibilidad, fundamentalmente, no se ha podido abordar un estudio cualitativo paralelo con entrevistas dirigidas a los consumidores de productos agroecológicos que suelen comprar en el Mercado Fresco de Mérida (estando representados por una población eminentemente extranjera), como han aportado muchos de nuestros informantes y nosotros mismos hemos contrastado in situ en las dos jornadas semanales que se celebraba el mercado. Este hecho nos hubiera aportado una información complementaria a la obtenida y, así, poder contrastar el punto de vista del productor con el del consumidor.

Con respecto a futuras líneas de investigación, lo mencionado podría ser un trabajo a realizar, además de la posibilidad de estudiar experiencias ya desarrolladas de este tipo en algún país europeo con una trayectoria más dilatada en este tipo de producciones y mercados y una revisión bibliográfica sobre estas, que nos sirvieran de referencia a la hora de extraer unas conclusiones a nivel comparativo. 


\section{Referencias bibliográficas}

Aldrich, Howard (1999). Organizations Evolving. U. K.: SAGE.

Andrews, Geoff (2008). The Slow Food Story: Politics and Pleasure. London: Pluto Press.

BacQ, Sophie y Janssen, Frank (2011). "The multiple faces of social entrepreneurship: a review of definitional issues based on geographical and thematic criteria". Entrepreneurship \& Regional Development: An International Journal, 23 (5-6), 373-403.

Boisier, Sergio (2003).“'Y si el desarrollo fuese una emergencia sistémica?» Revista del Centro Latinoamericano de Administración para el Desarrollo, Reforma y Democracia, 27, 1-24. Caracas.

Bruyat, Christian (1993). Creation d'entreprise: contributions epistemologiques et modetisation (tesis doctoral). France: Université Perre Mendès France (Grenoble II). Ecole Supérieure des Affaires.

Clamp, Christina A. y Alhamis, Innocentus (2010). «Social Entrepreneurship in the Mondragon Co-operative Corporation and the Challenges of Successful Replication". Journal of Entrepreneurship, 19 (2), 149-177.

Davies, John, Hides, Mick y Powell, James (2002)."Defining the development needs of entrepreneurs in SMEs”. Education E Training, 44 (8-9), 406-412.

Diario de Yucatán (29 de enero de 2017). «Del árbol a la mesa. El movimiento Slow Food gana adeptos cada día».

FILIon, Louis Jacques (2001). Entrepreneurs et propriétaires-dirigeants de PME. École des Hautes Études Commerciales (HEC), Montreal. Cahier de recherche no. 2001-03. ISSN: 0840-853X.

Izacara Palacios, Simón Pedro (2014). Manual de investigación cualitativa. México D. F.: Fontamara, SA

Kantis, Hugo, Ishida, Masahiko y Komori, Masahiko (2002). Entrepreneurship in emerging economies: the creation and development of new firms in Latin America and East Asia. Inter-American Development Bank. Disponible en: <http://www.iadb.org/sds/doc/idbenglishbookfinal.pdf>.

Kantis, Hugo, Ishida, Masahiko y Komori, Masahiko (2002). Empresarialidad en economías emergentes: creación y desarrollo de nuevas empresas en América Latina y el Este de Asia. Banco Interamericano de Desarrollo. Disponible en: <http://www.iadb.org/sds/doc/idbspanishbookfinal.pdf $>$.

Klofsten, Magnus (2000). "Training entrepreneurship at universities: a Swedish case". Journal of European Industrial Training, 24 (6), 337-344. 
Lотті, Ariane (2008). “The commoditization of products and taste: Slow Food and the conservation of agrobiodiversity". Agric Hum Values, 27:71-83. DOI: 10.1007/s10460-009-9213-x.

Mосні, Prudencio (2006). "Globalización, desarrollo local y descentralización. La importancia del conocimiento y la formación de recursos humanos en estos contextos". En Cristina Girardo, María de Ibarrola, Claudia Jacinto y Prudencio Mochi (coords.) (2006). Estrategias educativas y formativas para la inserción social y productiva. (pp. 145-161). Montevideo: OIT/Cinterfor, UNESCO.

Ortí, Alfonso (2000). "La apertura y el enfoque cualitativo o estructural: La entrevista abierta semidirectiva y la discusión de grupo". En M. A. García Ferrando, J. Ibáñez y F. Alvira (comps.). El análisis de la realidad social. Métodos y técnicas de investigación. Madrid: Alianza Universidad.

Riojas, Carlos (2007). "Estrategias de desarrollo regional en México y Europa Central. En busca de nuevas dimensiones institucionales en el ocaso de la era neoliberal". Argumentos. Estudios críticos de la sociedad, 20 (55), 13-47.

Sassatelli, Roberta y Davolio, Federica (2010). "Consumption, pleasure and politics. Slow Food and the político-aesthetic problematization of food". Journal of Consumer Culture, 10 (2), 202-232. DOI: 10.1177/1469540510364591.

Seale, Clive (2002). "Quality issues in qualitative inquiry." Qualitative Social Work, 1 (1), 97-110.

Smallbone, David (2011). "Políticas de espíritu empresarial y desarrollo de pyme, con referencia en particular a economías de transición". En Rodrigo Varela (ed.). Desarrollo, Innovación y Cultura Empresarial, vol. 3 (pp. 15-43). Colombia: Universidad ICESI.

TAYlor, Steven J. y Bogdan, Robert (2000). Introducción a los métodos cualitativos de investigación: la búsqueda de significados. Barcelona: Paidós.

Tencati, Antonio y Zsolnai, Lazslo (2012). "Collaborative Enterprise and Sustainability: The Case of Slow Food". Journal of Business Ethics, 110 (3), 345-354. DOI: 10.1007/s10551-011-1178-1.

Ucbasaran, Deniz, Gestead, Paul y Wright, Mike (2001). "The focus of entrepreneurial research. Contextual and process issues." Entrepreneurship theory and practice, 25 (4), 57-80.

Van Bommel, Koen y Spicer, André (2011). "Hail the Snail: Hegemonic Struggles in the Slow Food Movement". Organization Studies, 32 (12), 1717 1744. DOI: 10.1177/0170840611425722. 